\title{
Neutrino spin oscillations in magnetized moving and polarized matter
}

\author{
Umsalimat Abdullaeva, ${ }^{a}$ Vadim Shakhov, ${ }^{a, *}$ Alexander Studenikin ${ }^{a, b}$ and Alexander \\ Tsvirov $^{a}$ \\ ${ }^{a}$ Faculty of Physics, Lomonosov Moscow State University, \\ Moscow 119991, Russia \\ ${ }^{b}$ Joint Institute for Nuclear Research, \\ Dubna 141980, Moscow Region, Russia \\ E-mail: Abdullaeva.um@gmail.com, schakhov.vv15@physics.msu.ru, \\ studenik@srd.sinp.msu.ru, aleksander.tsvirov@yandex.ru
}

We present an analysis of the neutrino spin oscillations in magnetized moving and polarized matter. In this contribution, a generalization is made in respect to our previous studies [1,2] and the effects of the transversal magnetic field, transversal motion and polarization of matter are simultaneously accounted for. The cases of Dirac and Majorana neutrinos are considered.

*** The European Physical Society Conference on High Energy Physics (EPS-HEP2021), ***

*** 26-30 July $2021 * * *$

*** Online conference, jointly organized by Universität Hamburg and the research center DESY ***

${ }^{*}$ Speaker 


\section{Introduction}

For many years, until 2004, it was believed that a neutrino helicity precession and the corresponding spin oscillations can be induced by the neutrino magnetic interactions with an external electromagnetic field that provided the existence of the transversal magnetic field component $B_{\perp}$ in the particles rest frame. A new and very interesting possibility for neutrino spin (and spin-flavour) oscillations engendered by the neutrino interaction with matter background was proposed and investigated for first time in [3]. It was shown [3] that neutrino spin oscillations can be induced not only by the neutrino interaction with a magnetic field, as it was believed before, but also by neutrino interactions with matter in the case when there is a transversal matter current or matter polarization.

For historical notes reviewing studies of the discussed effect see in [4-6]. Note that the existence of the discussed effect of neutrino spin oscillations engendered by the transversal matter current and matter polarization and its possible impact in astrophysics have been confirmed in a series of papers [7-10].

In our recent papers $[1,2]$ we have developed a consistent quantum treatment of the neutrino spin and spinflavor oscillations engendered by the transversal matter currents. The presence of the transversal and longitudinal magnetic fields as well as the longitudinal matter currents are accounted for. Here below [11] we consider the quantum treatment to take into account the matter polarization in neutrino oscillations. Also [12] we consider the case of Majorana neutrino.

\section{Neutrino spin oscillations $v_{e}^{L} \Leftarrow\left(j_{\perp}, \zeta_{\perp}\right) \Rightarrow v_{e}^{R}$ engendered by transversal matter currents and transversal matter polarization: quantum treatment}

Consider two flavour Dirac neutrinos with two possible helicities $v_{f}=\left(v_{e}^{+}, v_{e}^{-}, v_{\mu}^{+}, v_{\mu}^{-}\right)^{T}$ in moving matter composed of electrons. Also we consider that neutrino moves along electron current and the polarization of the neutrino directed against the magnetic field direction. The neutrino interaction Hamiltonian reads

$$
H_{\text {int }}=\Delta H_{\text {weak }}^{\text {eff }}+\Delta H_{\zeta}^{e f f}=f^{\mu} \sum_{l} \bar{v}_{l}(x) \gamma_{\mu} \frac{1-\gamma_{5}}{2} v_{l}(x)=f^{\mu} \sum_{i} \bar{v}_{i}(x) \gamma_{\mu} \frac{1-\gamma_{5}}{2} v_{i}(x),
$$

where $f^{\mu}=\frac{G_{F}}{2 \sqrt{2}}\left(\left(1+4 \sin ^{2} \theta_{W}\right) j_{e}^{\mu}-\lambda_{e}^{\mu}\right), j_{e}^{\mu}=n_{0}(1, \mathbf{v})-$ matter current, $\lambda_{e}^{\mu}=n\left(\left(\zeta_{e}, \mathbf{v}_{e}\right), \zeta_{e} \sqrt{1-v_{e}^{2}}+\right.$ $\left.\mathbf{v}_{\mathbf{e}} \frac{\left(\zeta_{\mathbf{e}} \mathbf{v}_{\mathbf{e}}\right)}{1+\sqrt{1-\mathbf{v}_{\mathbf{e}}^{2}}}\right)$ - polarization vector, $l=e, \mu$ indicates the neutrino flavour and $i=1,2$ indicates the neutrino mass state. Each of the flavour neutrinos is a superposition of the neutrino mass states,

$$
v_{e}^{ \pm}=v_{1}^{ \pm} \cos \theta+v_{2}^{ \pm} \sin \theta, \quad v_{\mu}^{ \pm}=-v_{1}^{ \pm} \sin \theta+v_{2}^{ \pm} \cos \theta .
$$

Using the exact free neutrino mass states spinors,

$$
v_{\alpha}^{s}=C_{\alpha}\left(\begin{array}{c}
u_{\alpha}^{s} \\
\frac{\sigma p_{\alpha}}{E_{\alpha}+m_{\alpha}} u_{\alpha}^{s}
\end{array}\right) \sqrt{\frac{\left(E_{\alpha}+m_{\alpha}\right)}{2 E_{\alpha}}} \exp \left(i \boldsymbol{p}_{\alpha} \boldsymbol{x}\right)
$$

where the two-component spinors define neutrino helicity states, and are given by $u_{\alpha}^{+}=\left(\begin{array}{ll}1 & 0\end{array}\right)^{T}$, $u_{\alpha}^{-}=\left(\begin{array}{ll}0 & 1\end{array}\right)^{T}$, for the typical term $\Delta_{\alpha \alpha^{\prime}}^{s s^{\prime}}=\left\langle v_{\alpha}^{s}\left|\Delta H_{\zeta}^{e f f}\right| v_{\alpha^{\prime}}^{s^{\prime}}\right\rangle$, which by fixing proper values of 
$\alpha, s, \alpha^{\prime}$ and $s^{\prime}$ can reproduce all of the elements of the neutrino evolution Hamiltonian $\Delta H_{\zeta}^{e f f}$ that accounts for the effect of matter polarization, we obtain,

$$
\Delta_{\alpha \alpha^{\prime}}^{s s^{\prime}}=G n\left\{u_{\alpha}^{s T}\left[\left(\begin{array}{ll}
0 & 0 \\
0 & 2
\end{array}\right) \zeta_{\|}+\left(\begin{array}{cc}
0 & \gamma_{\alpha}^{-1} \\
\gamma_{\alpha^{\prime}}^{-1} & 0
\end{array}\right) \zeta_{\perp}\right] u_{\alpha^{\prime}}^{s^{\prime}}\right\} \delta_{\alpha}^{\alpha^{\prime}}
$$

where $\zeta_{\|}$and $\zeta_{\perp}$ are the longitudinal and transversal polarization of the matter, $G=\frac{G_{F}}{2 \sqrt{2}}, n=\frac{n_{0}}{\sqrt{1-v^{2}}}$ and

$$
\gamma_{\alpha \alpha^{\prime}}^{-1}=\frac{1}{2}\left(\gamma_{\alpha}^{-1}+\gamma_{\alpha^{\prime}}^{-1}\right), \quad \tilde{\gamma}_{\alpha \alpha^{\prime}}^{-1}=\frac{1}{2}\left(\gamma_{\alpha}^{-1}-\gamma_{\alpha^{\prime}}^{-1}\right), \quad \gamma_{\alpha}^{-1}=\frac{m_{\alpha}}{E_{\alpha}} .
$$

The effective interaction Hamiltonian in the flavour basis has the following structure,

$$
H_{\zeta}^{f}=n G\left(\begin{array}{cccc}
0 & \left(\frac{\eta}{\gamma}\right)_{e e} \zeta_{\perp} & 0 & \left(\frac{\eta}{\gamma}\right)_{e \mu} \zeta_{\perp} \\
\left(\frac{\eta}{\gamma}\right)_{e e} \zeta_{\perp} & 2 \zeta_{\|} & \left(\frac{\eta}{\gamma}\right)_{e \mu} \zeta_{\perp} & 0 \\
0 & \left(\frac{\eta}{\gamma}\right)_{e \mu} \zeta_{\perp} & 0 & \left(\frac{\eta}{\gamma}\right)_{\mu \mu} \zeta_{\perp} \\
\left(\frac{\eta}{\gamma}\right)_{e \mu} \zeta_{\perp} & 0 & \left(\frac{\eta}{\gamma}\right)_{\mu \mu} \zeta_{\perp} & 2 \zeta_{\|}
\end{array}\right)
$$

where we use the following notations:

$$
\left(\frac{\eta}{\gamma}\right)_{e e}=\frac{\cos ^{2} \theta}{\gamma_{11}}+\frac{\sin ^{2} \theta}{\gamma_{22}}, \quad\left(\frac{\eta}{\gamma}\right)_{\mu \mu}=\frac{\sin ^{2} \theta}{\gamma_{11}}+\frac{\cos ^{2} \theta}{\gamma_{22}}, \quad\left(\frac{\eta}{\gamma}\right)_{e \mu}=\frac{\sin 2 \theta}{\tilde{\gamma}_{21}} .
$$

For the oscillation $v_{e}^{L} \Leftarrow\left(j_{\perp}, \zeta_{\perp}, B_{\perp}\right) \Rightarrow v_{e}^{R}$ probability we get

$$
P_{v_{e}^{L} \rightarrow v_{e}^{R}}(x)=\sin ^{2} 2 \theta_{\mathrm{eff}} \sin ^{2} \frac{\pi x}{L_{\mathrm{eff}}}, \quad \sin ^{2} 2 \theta_{\mathrm{eff}}=\frac{E_{\mathrm{eff}}^{2}}{E_{\mathrm{eff}}^{2}+\Delta_{\mathrm{eff}}^{2}}, \quad L_{\mathrm{eff}}=\frac{\pi}{\sqrt{E_{\mathrm{eff}}^{2}+\Delta_{\mathrm{eff}}^{2}}},
$$

where

$$
E_{\mathrm{eff}}=\left|\mu_{e e} B_{\perp}-\left(\frac{\eta}{\gamma}\right)_{e e} n\left[\widetilde{G} v_{\perp}+G \zeta_{\perp}\right]\right|, \Delta_{\mathrm{eff}}=\left|\left(\frac{\mu}{\gamma}\right)_{e e} B_{\|}+n\left[\widetilde{G}\left(1-v_{\|}\right)+G \zeta_{\|}\right]\right|,
$$

and $\tilde{G}=\frac{G_{F}}{2 \sqrt{2}}\left(1+4 \sin ^{2} \theta_{W}\right)$. Here $B_{\|}, B_{\perp}, v_{\|}$and $v_{\perp}$ are the longitudinal and transversal components of the external magnetic field $\boldsymbol{B}$ and matter velocity $\boldsymbol{v}$ and

$$
\mu_{e e}=\mu_{11} \cos ^{2} \theta+\mu_{22} \sin ^{2} \theta+\mu_{12} \sin 2 \theta, \quad\left(\frac{\mu}{\gamma}\right)_{e e}=\frac{\mu_{11}}{\gamma_{11}} \cos ^{2} \theta+\frac{\mu_{22}}{\gamma_{22}} \sin ^{2} \theta+\frac{\mu_{12}}{\gamma_{12}} \sin 2 \theta .
$$

These formulas can be useful in further investigations of neutrino oscillations in extreme astrophysical environments.

\section{The case of Majorana neutrino}

Now we consider the case of a Majorana neutrino that propagates in a moving media composed of neutrons in the presence of a magnetic field. In the mass basis for Majorana neutrinos the magnetic moment has only nondiagonal components, so that $\mu_{11}=\mu_{22}=0, \mu_{12}=\mu_{21}^{*} \equiv i \mu$, and $\mu$ is real. 
In the case of unpolarized matter the corresponding oscillation probability $P_{v_{e}^{L} \rightarrow v_{\mu}^{R}}$ is determined by (8) with

$$
\begin{aligned}
& E_{\mathrm{eff}}=\sqrt{\left(\mu B_{\perp} \sin \phi \cos 2 \theta+2\left(\frac{\eta}{\gamma}\right)_{e \mu} \tilde{G} n v_{\perp}\right)^{2}+\left(\mu B_{\perp} \cos \phi \cos 2 \theta\right)^{2}} \\
& \Delta_{\mathrm{eff}}=\left|\frac{\Delta m^{2} \cos 2 \theta}{4 p_{0}^{v}}-2 \tilde{G} n\left(1-v_{\|}\right)\right|
\end{aligned}
$$

where $\Delta M=\frac{\Delta m^{2} \cos 2 \theta}{4 p_{0}^{\gamma}}, \phi$ is an angle between $\mathbf{B}_{\perp}$ and $\mathbf{v}_{\perp},\left(\frac{\mu}{\gamma}\right)_{e \mu}=\frac{\mu_{12}}{\gamma_{12}} \cos 2 \theta+\frac{1}{2}\left(\frac{\mu_{22}}{\gamma_{22}}-\frac{\mu_{11}}{\gamma_{11}}\right) \sin 2 \theta$.

From the comparison of the oscillation probabilities of Majorana and Dirac neutrinos (see also [12]) it follows that Majorana and Dirac neutrinos exhibit quite different oscillation patterns. In particular, the efficiency of mixing is quite different in these two cases, and also the resonance conditions in these two cases are realized at different densities of the matter.

\section{Acknowledgments}

The work is supported by the Interdisciplinary Scientific and Educational School of Moscow University "Fundamental and Applied Space Research" and by the Russian Foundation for Basic Research under grant No. 20-52-53022-GFEN-a.

\section{References}

[1] P. Pustoshny, V. Shakhov and A. Studenikin, PoS EPS-HEP2019, 429 (2020).

[2] P. Pustoshny and A. Studenikin, Neutrino spin and spin-flavour oscillations in transversal matter currents with standard and non-standard interactions, Phys. Rev. D 98, 113009 (2018).

[3] A. I. Studenikin, Phys. Atom. Nucl. 67, 993 (2004).

[4] A. Studenikin, J. Phys. Conf. Ser. 888, 012221 (2017).

[5] A. Studenikin, J. Phys. Conf. Ser. 718, 062076 (2016).

[6] A. Studenikin, PoS NOW2016, 070 (2017).

[7] V. Cirigliano, G. M. Fuller and A. Vlasenko, Phys. Lett. B 747, 27 (2015).

[8] C. Volpe, Int. J. Mod. Phys. E 24, 1541009 (2015).

[9] A. Kartavtsev, G. Raffelt and H. Vogel, Phys. Rev. D 91, 125020 (2015).

[10] A. Dobrynina, A. Kartavtsev and G. Raffelt, Phys. Rev. D 93, 125030 (2016).

[11] A. Tsvirov, Bachelor Dissertation "Neutrino motions and oscillations in matter and magnetic field", Moscow State University, Moscow, 2021.

[12] U. Abdullaeva, Bachelor Dissertation "Majorana neutrino in moving matter and magnetic field", Moscow State University, Moscow, 2020. 